Nervenarzt 2009 $\cdot 80: 513-514$

DOI 10.1007/s00115-009-2783-4

Online publiziert: 24. April 2009

(c) Springer Medizin Verlag 2009

\author{
H.-J. Möller \\ Klinik für Psychiatrie und Psychotherapie, Ludwig-Maximilian-Universität, München
}

\title{
Unipolare depressive Erkrankungen
}

der Depression zusammen, wobei insbesondere die vorrangig durch somatische Beschwerden geprägte Depression häufig verkannt wird. Ein weiteres, zur Unterdiagnostizierung führendes Problem ist, dass dieselben Menschen, die an depressiven Symptomen leiden, die Krankheit nicht erkennen und deshalb keine ärztliche Hilfe aufsuchen. Auch die Angst vor Stigmatisierung spielt in dem Zusammenhang eine wichtige Rolle. Nicht erkannte und nicht behandelte Depressionen stellen ein großes Problem dar, da die Depression eine Erkrankung ist, die mit hohem Leid und hoher Belastung der Betroffenen und der Familienangehörigen einhergeht und die durch Arbeitsunfähigkeit und ggf. frühzeitige Berentung hohe ökonomische Konsequenzen hat. Nicht zuletzt sei daran erinnert, dass die Depression wegen des hohen Risikos suizidalen Verhaltens prinzipiell als tödliche Erkrankung eingeschätzt werden muss.

\section{(7) Auch heute noch wird die Depression häufig zu spät diagnostiziert}

In der Behandlung der Depression, insbesondere der mittelgradigen und schweren Depressionsformen, spielt die medikamentöse Behandlung mit Antidepressiva eine wichtige Rolle. Sie wird zu einem großen Teil von Hausärzten durchgeführt. Patienten mit schweren und komplikationsreicheren Depressionsformen werden meistens durch Psychiater, ambulant oder stationär, behandelt. Die Behandlung mit Antidepressiva ist trotz kritischer Hinterfragung der Wirksamkeit der Antidepressivatherapie im Rahmen von metaanalytischen Auswertungen als durchaus klinisch relevant einzuschätzen. Sowohl die
Akut- als auch die Erhaltungstherapien erreichen mit einer NNT („number needed to treat") von 6 für die Akuttherapie und 4 für die Erhaltungstherapie eine Größenordnung, die im Allgemeinen als mittelgradig, als vergleichbar mit vielen internistischen Therapien angesehen werden kann [1]. Auch die in den letzten Jahren intensiv diskutierte Frage, ob die selektiven Serotoninwiederaufnahmehemmer oder überhaupt alle Antidepressiva neben einem die Suizidalität reduzierenden Effekt auch einen Suizidalität induzierenden Effekt bei bestimmten Risikopatienten und unter bestimmten Bedingungen haben kann, sollte keinesfalls dazu führen, einem Patienten die Behandlung mit Antidepressiva vorzuenthalten [2]. Um die Wirksamkeit von Antidepressiva zu optimieren, ist es wichtig, die Entscheidung für eine bestimmte medikamentöse Therapie individuell anzupassen. Dazu können die Berücksichtigung von Subtypen der depressiven Erkrankung sowie auf den Einzelfall bezogene klinische Charakteristika und in Zukunft auch ganz besonders pharmakogenetische Parameter beitragen. Dies gilt sowohl für Wirksamkeitsangaben als auch im Hinblick auf Nebenwirkungen.

\section{(?) Die Entscheidung für ein bestimmtes Antidepressivum ist individuell zu treffen}

Während früher die Therapie der unipolaren Depressionen vorrangig als Therapie mit Antidepressiva angesehen wurde, kombiniert natürlich immer mit supportiven Zwiegesprächen und ggf. sozialpsychiatrischen Angeboten, hat sich in den letzten Jahren zunehmend die Psychotherapie eine immer größere Bedeu- 
tung erobert. Im Vergleich zu früher haben in der deutschen Psychiatrie diesbezüglich die pragmatisch einsetzbaren und individuell anpassbaren Verfahren aus dem Bereich der verhaltenstherapeutischen/kognitiven Therapie den psychodynamischen Methoden immer mehr den Rang abgelaufen. Psychotherapeutische Verfahren können, u. a. bei leichten Depressionen und zum Teil auch bei mittelschweren, als Monotherapie oder in Kombination mit Antidepressiva eingesetzt werden, insbesondere bei Depressionen mit erkennbaren psychologischen/biographischen Auslösern. Insbesondere unter bestimmten Bedingungen, $u$. a. bei chronifizierter Depression, ist eine Kombinationstherapie von antidepressiver Medikation und psychotherapeutischer Behandlung sinnvoll.

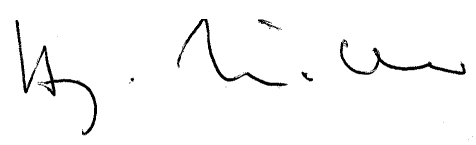

Prof. Dr. Hans-Jürgen Möller

\section{Korrespondenzadresse}

\section{Prof. Dr. H.-J. Möller}

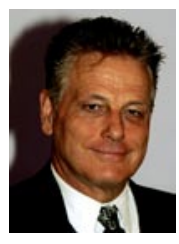

Klinik für Psychiatrie

und Psychotherapie,

Ludwig-Maximilian-Universität

Nussbaumstraße 7,

80336 München

Hans-Juergen.Moeller@

med.uni-muenchen.de

Interessenkonflikt. Der korrespondierende Autor weist auf folgende Beziehungen hin: Von den folgenden Firmen hat Herr Professor Möller Forschungsgelder erhalten, ist Mitglied des Advisory Boards oder aber erhält Honorare für Vorträge: AstraZeneca, Bristol-Myers Squibb, Eisai, Eli Lilly, GlaxoSmithKline, Janssen Cilag, Lundbeckd, Merck, Novartis, Organon, Pfizer, Sanofi-Aventis, Sepracor, Servier and Wyeth.

\section{Literatur}

1. Möller HJ (2008) Isn't the efficacy of antidepressants clinically relevant? A critical comment on the results of the metaanalysis by Kirsch et al 2008. Eur Arch Psych Clin Neurosci 258:451-455

2. Möller HJ, Baldwin D, Goodwin G et al (2008) Do SSRIs or antidepressants in general increase suicidality? WPA-Section on Pharmacopsychiatry: consensus statement. Eur Arch Psych Clin Neurosci 258:3-23

3. Hegerl U, Plattner A, Möller HJ (2004) Should combined pharmaco- and psychotherapy be offered to depressed patients? A qualitative review of randomized clinical trials from the 1990s. Eur Arch Psychiatry Clin Neurosci 254(2):99-107

\section{Neue SHARE-Daten vermitteln Einblicke in Alterungsprozesse}

Die Daten der zweiten SHARE-Erhebungswelle sind ab sofort für Wissenschaftler kostenlos downzuloaden. SHARE trägt damit der Aufforderung des Europäischen Rats Rechnung, eine systematische Datenbasis für die empirische Alterungsforschung in Europa aufzubauen.

SHARE - Der "Survey of Health, Ageing and Retirement in Europe" ist ein Projekt, das durch Umfragen einen interdisziplinären Paneldatensatz zu den Themen Gesundheit, wirtschaftliche Situation und zu sozialen und familiären Beziehungen von Menschen ab einem Alter von 50 Jahren erstellt. Umfragedaten von mehr als 40.000 Personen aus bis zu 15 verschiedenen europäischen Ländern sind darin enthalten.

Die Daten werden in mehreren Erhebungswellen generiert.

SHARE bietet Informationen, wie individuelle und gesellschaftsumfassende Alterungsprozesse in den verschiedenen Ländern durch die politische, wirtschaftliche, soziale und kulturelle Vielfalt beeinflusst werden. Basierend auf den Daten der ersten Umfrage wurden von über 1000 Wissenschaftlern bereits eine Vielzahl an Erkenntnissen gewonnen.

Die Daten der zweiten Erhebungswelle sind ab sofort für Wissenschaftler weltweit kostenlos unter www.share-project.org erhältlich.

Erste Ergebnisse im Themengebiet "Gesundheit" zeigen, Südeuropäer leben länger, obwohl es Nordeuropäern gesundheitlich und finanziell besser geht. Außerdem leiden Menschen mit niedrigerem Einkommen oder Vermögen in allen Ländern, vor allem aber in Nordeuropa häufiger unter Depressionen. Ebenso ist die geriatrische Pflege verbesserungsbedürftig.

Im Bereich „Beschäftigung" konnten die bisherigen SHARE-Daten zeigen, dass sich Maßnahmen zur Gesundheitsprävention am Arbeitsplatz lohnen. So gehen Gesunde zwei Jahre später als gesundheitlich eingeschränkte in Rente. Schon eine Investition von $3 \%$ des Arbeitseinkommens in die gesundheitliche Vorsorge reicht aus, um die Arbeitsfähigkeit zu erhalten. Ebenso fördern gute Arbeitsplatzbedingungen einen späteren Renteneintritt.

Auf dem Gebiet „familiärer und sozialer Netzwerke" beweisen die SHARE-Daten, dass der Bedeutungsverlust der Familie ein Mythos ist. In ganz Europa ist die gegenseitige Unterstützung weit verbreitet. Bei den über 65-Jährigen verbringt ein Drittel durchschnittlich fast fünf Stunden damit auf Enkelkinder aufzupassen oder Familienmitglieder in anderer Art zu unterstützen. Interessanterweise ist die Richtung der finanziellen Unterstützung der Familie in Europa divergent. Im Norden sind es die Eltern, im Süden die erwachsenen Kinder, die finanziell unter die Arme greifen. Die neuen Daten werden weitere Einblicke zu diesen Themen in Europa liefern. SHARE wird durch die Europäische Kommission, nationale Geldgeber der teilnehmenden Länder und durch das National Institute on Aging finanziert.

Quelle: Universität Mannheim, http://www.uni-mannheim.de/ 